
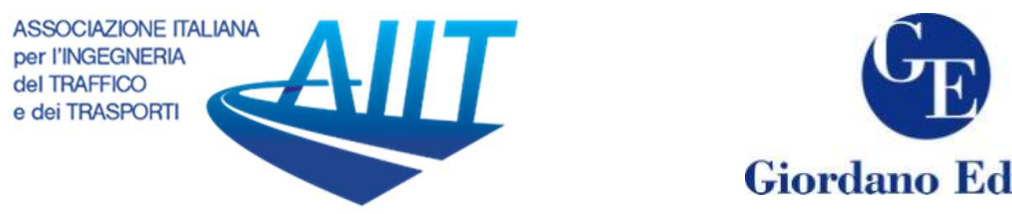

Giordano Editore

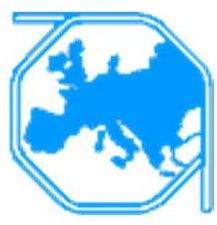

\title{
Fitting Volume Delay Functions under interrupted and uninterrupted flow conditions at Greek urban roads
}

\author{
Roza E. Barka ${ }^{1 *}$, Ioannis Politis ${ }^{2}$ \\ ${ }^{1}$ PhD Candidate, ${ }^{2}$ Assistant Professor \\ Laboratory of Transportation Engineering \\ Department of Civil Engineering \\ Aristotle University of Thessaloniki, Greece
}

\begin{abstract}
This paper presents the calibration of the most commonly used Volume Delay Functions (VDF): BPR, Conical, Akcelik and Modified Davidson, for an urban area populated by over 1 million inhabitants, the city of Thessaloniki in Greece. The estimation of the unknown coefficients was carried out for a typical freeway, the ring road of the city, and selected arterial and collected roads of the city center, through recent data of hourly observed vehicle speeds and volumes obtained from video recordings and loop detectors. The BPR function yielded the highest accuracy across all the examined road sections and was characterized as the most suitable to simulate and interpret the existing traffic conditions. The estimated coefficients differed significantly from the values proposed in the pertinent literature, which highlights the importance of using locally derived data for the calibration of the VDFs.
\end{abstract}

Keywords: Volume Delay Functions, Trip Assignment, Speed Forecasting, Congestion Functions, Travel Demand Models

\section{Introduction}

Travel demand models in the transportation planning process consist of four distinctive steps: trip generation, trip distribution, mode choice and traffic assignment (Huntsinger and Rouphail, 2011; Subbarao and Krishnarao, 2016), though this sequence is not the only one followed by planners (Marquez, 2014). During the last stage, traffic assignment (Dhulipala, Kedia and Katti, 2020), the identification of future transport needs is completed, which is vital to achieve congestion control and for the rational exploitation of the road infrastructure (He and Zao, 2013). Volume delay functions have been widely applied in macroscopic traffic assignment (Kucharski and Drabicki, 2017) to define travel speed (or travel time) as a function of traffic volume. For low volumes, the travel speed is similar to the free flow speed, while as the volumes reach the capacity of the link the rate of decrease becomes greater, resulting in a sharp decline in speed (Mtoi and Moses, 2014).

* Corresponding author: Roza E. Barka (rozampar@civil.auth.gr) 
The accurate forecasting of speed is essential for the development of models concerning route allocation in transportation planning (Mtoi and Moses, 2014), as well as for the evaluation of the level of service in road sections, the economic evaluation of road projects and the possible introduction of safety measures (Seetharaman et al., 2011). Despite the fact that travel time and delay are often more intuitively perceived by the user (Chepuri et al., 2018), speeds are widely used to determine fuel consumption and emissions from vehicles (Rose, 1994). Moreover, the impact of differences in geometrical and operational characteristics of road sections on operating speeds can directly be evaluated through volume delay functions (Stevens, Barkley and Miller, 2017).

According to Martin and McGuckin (1998) three different approaches can be applied when estimating the form of volume delay functions:

1.A single type regardless of the facility type

2.Different types depending on the facility type

3.Different types depending on the facility type and the estimated delay at intersections

The second approach has been proved to improve the accuracy of the estimated functions, while the use of locally derived data is needed for a better representation of the local conditions (Rose, Taylor and Tisato, 1989) that differ due to varying demographic, economic and drivers' behavioral data (Suh, Park and Kim, 1990). Recent data are needed, since changes in infrastructure, population and speed limits affect the function's ability to represent current conditions.

The main aim of the following study is the calibration of the most commonly used volume delay functions: BPR, Akcelik, Conical and Modified Davidson, for selected urban sections of the city center and for the Ring Road of Thessaloniki (typical freeway) in Greece, through data of hourly observed pairs of speeds and volumes that were obtained through loop detectors and video processing installations. Given that the last published research in the area of Thessaloniki took place twenty years ago and focused solely on the calibration of the BPR function, recent locally collected data were used to update the input parameters of the BPR function and to estimate the remaining VDFs for the region. The root mean square error (RMSE) and the coefficient of determination $\left(\mathrm{R}^{2}\right)$ were used for the evaluation of the derived functions, as well as for the selection of an appropriate VDF for each road section. Road sections were divided according to their facility type and grouped by the estimated free flow speed, in an attempt to improve the model's accuracy.

The paper is organized as follows. Literature is reviewed in Section 2, where the estimated parameters of various researches are presented and the drawbacks of each VDF are explained. Subsequently, Section 3 focuses on the data acquisition and processing for both interrupted and uninterrupted road sections, while the estimation of the VDFs takes place in Section 4. In Section 5 we present the results of the study, combined with the evaluation of the derived VDFs. Finally, the conclusions and recommendations are presented (Section 6).

\section{Literature Review}

\subsection{Bureau of Public Roads}

The typical BPR function, developed by the Bureau of Public Roads at the United States, is perhaps the most commonly used VDF in literature, due to its simplicity. In physical terms, $a$ represents the ratio of travel speed per unit distance at practical capacity 
to that at free-flow, while $b$ represents the rate of decrease in speed as congested conditions are reached (Moses et al., 2013). The BPR function is presented below:

$$
u=\frac{u o}{\left(1+a * x^{b}\right)}
$$

where $u=$ travel speed at road section, $u o=$ free-flow speed, $a, b=$ unknown parameters, $x=V / C$ ratio

Despite its simple mathematical form, BPR has a number of limitations. It has been proved that the function tends to underestimate travel speeds for $v / c$ ratios of less than 1 and to overestimate them for $v / c$ ratios higher than 1 (Huntsinger and Rouphail, 2011; Skabardonis and Dowling, 1997).

Since its initial development, multiple attempts have been made by planners in a number of countries to specify the values of $a$ and $b$ for the BPR function. Nielsen and Jørgensen (2008) claimed that for Danish road sections $a$ ranges between 0.8 and 1.2, according to the road type, while $b$ increases from 1.5 (two-lane roads) to 4 (multi-lane motorways). Martin and McGuckin (1998) recommended values of $a=0.84$ and $b=5.5$ for small urban areas, while for Korean highways values of $a=2.72$ and $b=6$ were found to be the best fit (Suh, Park and Kim, 1990).

Nobel and Yagi (2017) investigated the ability of the typical BPR $(a=0.15, b=4)$ to represent the local conditions in Manila, Philippines, and concluded that the estimated errors declined noticeably, when values of $a=0.5$ and $b=1.5$ were introduced. Similarly, a comparison between the typical BPR and the curves recommended by Highway Capacity Manual 94 (Skabardonis and Dowling, 1997) revealed that updated curves for interrupted and uninterrupted facilities improve the accuracy of the estimated travel speeds.

\subsection{Conical}

To overcome several limitations of the BPR curve, Spiess (1990) introduced the Conical delay function, which was derived from geometry and basic algebra. At the time, the computing time of the equilibrium process was minimized, when Conical function was used. The conical function is:

$$
u=\frac{u o}{\left(2-b *(1-x)-a+\sqrt{\left.b^{2} *(1-x)^{2}+a^{2}\right)}\right.}
$$

where $u=$ travel speed at road section, $u o=$ free-flow speed, $a, b=$ unknown parameters, $x=V / C$ ratio, while $a$ depends on $b$ :

$$
a=\frac{b-0,5}{b-1}
$$

Davis and Xiong (2007) proposed the values of $b=4$ for divided and 5 for undivided arterials, while $b$ needs to be higher than 1.1, in any case. In several studies (Marquez, 2014; Huntsinger and Rouphail, 2011), the estimation of the Conical function resulted in the highest errors among all functions. Its poor performance was attributed to the dependency between a and $b$, which made it difficult to reach a better adjustment.

\subsection{Akcelik}

Unlike the previous functions, the Akcelik function, proposed by Rahmi Akcelik, contains only one unknown parameter $j$ that needs to be estimated. Dowling, Singh and Cheng (1998) refer to the following values for the coefficient $J: 0,4$ for urban arterials, 
0,8 for collectors and 1,6 for local streets. In general, higher values of $J$ are proposed for arterial roads, whereas lower ones are suggested for freeways (Mtoi and Moses, 2014). The Akcelik function is shown below:

$$
u=\frac{u o}{\left(1+0,25 * u o *\left[(x-1)+\sqrt{(x-1)^{2}+\frac{8 * j * x}{c * T}}\right]\right)}
$$

where $u=$ travel speed at road section, $u o=$ free-flow speed, $x=V / C$ ratio, $j=$ unknown parameter, $c=$ capacity of road section, $T=$ analysis period.

The Akcelik function is considered to provide an accurate estimation of speed under congested conditions. However, in their study, Stevens, Barkley and Miller (2017) indicated that the estimated errors increase sharply, when large periods of analysis are preferred.

\subsection{Modified Davidson}

Initially, the Davidson function consisted of a simple form, that was unable to describe the traffic conditions when capacity was exceeded. The function was modified and a second part was added, while the division between the two parts is achieved through the unknown parameter $\mu$, ranging between 0.85 and 0.95 . The second unknown coefficient, $J$, depends on the land use of the area surrounding the road section and its facility type (Mtoi and Moses, 2014). The Modified Davidson function is:

$$
u= \begin{cases}\frac{u o}{\left(1+j * \frac{x}{(1-x)}\right)}, & x \leq \mu \\ \frac{v o}{\left(1+j * \frac{\mu}{(1-\mu)}+\frac{j *(x-\mu)}{(1-\mu)^{2}}\right)}, & x>\mu\end{cases}
$$

where $u=$ travel speed at road section, $u o=$ free-flow speed, $x=V / C$ ratio, $\mu, J=$ unknown parameters.

\subsection{Comparison between the functions in the literature}

In their study, Mtoi and Moses (2014) stated that each of the four mentioned VDFs is suitable for a certain facility type. Modified Davidson function presented remarkable performance, irrespective of the facility type, which was attributed to the flexibility of the coefficient $\mu$. Akcelik function yielded improved results for toll roads and signalized arterials, whereas updated BPR and Conical function were found to be more appropriate for uninterrupted facilities.

We are unaware of any other study concerning the evaluation and comparison of the four mentioned functions. However, Huntsinger and Rouphail (2011) used bottleneck and queue analysis to calibrate BPR, Akcelik and Conical function, through locally derived data concerning the I-40 in North Carolina. BPR yielded the highest accuracy, followed by Akcelik, while Conical underperformed. A similar comparison between BPR and Conical was conducted by Marquez (2014) for six multi-lane road sections in Bogota. The adjustment was significantly higher for the updated BPR function, which was found to be more suitable for the mentioned facility type. In their study, Oskarbski et al. (2017) estimated the unknown coefficients for Conical and BPR function across the Tri-City, including Gdansk, Sopot, Gdynia. Despite the fact that Conical function provided the best 
fit to the data, researchers considered that the results can not be used in traffic control, since the model should have led to higher accuracy.

\section{Method}

\subsection{Data Acquisition}

The study was based on field measurements from two different data sources. The calibration of the functions for the Ring Road of Thessaloniki was accomplished using detector data from 9 fixed traffic monitoring sites, provided by Trias S.A. The measurements used for this analysis consisted of hourly pairs of vehicle speeds and volumes (in vehicles per hour) and were collected during 10 consecutive months from May 2010 to February 2011, covering a total length of approximately $17 \mathrm{~km}$. For each month, measurements were available for a typical weekday and a typical Saturday, allowing for a better representation of the variation in speeds and traffic volumes.

The Transport Engineering Laboratory of the Aristotle University of Thessaloniki, Greece provided the necessary data for the central area of Thessaloniki. The data were measured throughout the year 2017 and consisted of hourly pairs of vehicle speeds and volumes, covering 48 consecutive hours (Barka, 2019). The data represented 6 roads of the city center (interrupted flow facilities): one main arterial, two secondary arterials and three main collectors. In this case, the traffic volumes had been previously grouped into 4 categories: motorcycles, passenger cars, buses and trucks. The volumes were, then, converted to equivalent passenger car units ( $\mathrm{pcu}$ ), using the corrective coefficients provided by the Ministry for the Environment, Physical Planning and Public Works (Antoniou and Spyropoulou, 2015). Table 1 illustrates the basic road characteristics for all the selected road sections, while the total number of records used for the calibration of the VDFs are illustrated in Table 2.

Table 1: The basic road characteristics for the selected road sections

\begin{tabular}{lccccc}
\hline Road Section & Area Type & Facility Type & $\begin{array}{c}\text { Number of Lanes } \\
\text { per }\end{array}$ & Wirection & of Lanes (m) \\
& & & $\begin{array}{c}\text { Gradient } \\
(\%)\end{array}$ \\
\hline Ring Road & Interurban & Uninterrupted & 3 & $3.1-3.5$ & - \\
Main Arterial & Urban & Interrupted & 3 & 3.00 & - \\
Secondary Arterial (Type 1) & Urban & Interrupted & 3 & 2.95 & - \\
Secondary Arterial (Type 2) & Urban & Interrupted & 3 & 3.00 & - \\
Main Collector (Type 1) & Urban & Interrupted & 4 & 3.00 & +3 \\
Main Collector (Type 2) & Urban & Interrupted & 4 & 3.50 & +5 \\
Main Collector (Type 3) & Urban & Interrupted & 2 & 3.00 & +10 \\
\hline
\end{tabular}

Table 2: Total number of records for each road section

\begin{tabular}{lc}
\hline Road Section & Total Number of Records \\
\hline Ring Road & 1056 \\
Main Arterial & 48 \\
Secondary Arterial (Type 1) & 32 \\
Secondary Arterial (Type 2) & 48 \\
Main Collector (Type 1) & 48 \\
Main Collector (Type 2) & 48 \\
Main Collector (Type 3) & 48 \\
\hline
\end{tabular}




\subsection{Estimation of free-flow speed}

The estimation of the free-flow speed for each road section was based on the definition of Highway Capacity Manual (2010), where free-flow speed is defined as the average running speed under low-volume conditions. However, given that the relationship between vehicle speed and volume is parabolic, according to the fundamental diagram, low-volume conditions can be observed both during free-flowing and congested conditions. To overcome this issue, we assumed that the free-flow speed should be representative of the low traffic volumes in the hypocritical part of the curve, for each road section.

By plotting the correlation between the observed speeds and the traffic volumes for the Ring Road of Thessaloniki, we concluded that the free-flow speed ranged from 80 to 110 $\mathrm{km} / \mathrm{h}$. As a result, the data were split into two categories in order to receive a representative free-flow speed for each data set. The estimation process yielded a freeflow speed of $95 \mathrm{~km} / \mathrm{h}$ and $105 \mathrm{~km} / \mathrm{h}$, for the first and second data set respectively. The same procedure was followed for the urban road sections. It should be noted that the two secondary arterials were not handled jointly, due to the fact that their free-flow speed differed significantly. The collectors were divided into two groups, based on their freeflow speed. Table 3 depicts the free-flow speed of each road section.

Table 3: Estimation of Free-Flow speed for each road section

\begin{tabular}{lc}
\hline Road Section & Free-Flow Speed $(\mathrm{km} / \mathrm{h})$ \\
\hline Ring Road (Type 1) & 95 \\
Ring Road (Type 2) & 105 \\
Main Arterial & 35 \\
Secondary Arterial (Type 1) & 55 \\
Secondary Arterial (Type 2) & 50 \\
Main Collectors (Group 1) & 45 \\
Main Collectors (Group 2) & 40 \\
\hline
\end{tabular}

\subsection{Estimation of Capacity}

The capacity of the Ring Road was assumed to be the $95^{\text {th }}$ percentile of the observed hourly vehicle flows, as opposed to the maximum hourly flow, in order to exclude outlier values (Mtoi and Moses, 2014). The estimation was conducted using the SPSS 23 software by IBM, for each data set separately. The calculation yielded $5528 \mathrm{veh} / \mathrm{h}$ for the data set with an estimated free-flow speed of $95 \mathrm{~km} / \mathrm{h}$ and $4544 \mathrm{veh} / \mathrm{h}$ for the remaining data set with an estimated free-flow speed of $105 \mathrm{~km} / \mathrm{h}$.

The estimation of the capacity for the urban road sections was conducted using the following equation (Dutta and Ahmed, 2019):

$$
c=s * g / C
$$

where $c=$ the capacity of each road section $(\mathrm{pcu} / \mathrm{h}), s=$ the saturation flow of each road section ( $\mathrm{pcu} / \mathrm{h}), C=$ the cycle time ( $\mathrm{sec}), g=$ the effective green time (sec).

The estimation of the saturation flow was based on the methodology proposed for road junctions controlled by traffic signals, by Kimber, McDonald and Hounsell (1986), to take into consideration all the factors that could affect the saturation flow of interrupted flow facilities. The saturation flow equation is presented below: 


$$
s=\frac{2080-140 * f n-42 * f g * G+100 *(w-3,25)}{1+1,5 P / R}
$$

Given that the pairs of vehicle speeds and volumes represented road sections and not road junctions, the effect of turning lanes was excluded from the analysis and the equation was simplified, as presented:

$$
s=2080-140 * f n-42 * f g * G+100 *(w-3,25)
$$

where $s=$ the saturation flow per lane ( $\mathrm{pcu} / \mathrm{h} / \mathrm{ln}), f n=$ a nearside lane dummy variable ( $=1$ for a nearside lane and zero otherwise), $f g=$ a gradient dummy variable $(=0$ for downhill entries and 1 for uphill entries), $G=$ the gradient of the road section (\%) $w=$ the lane width $(\mathrm{m})$. Finally, the effective green time for each road section was calculated using the following equation:

$$
g=k+a-l
$$

where $g=$ the effective green time (sec), $k=$ green light time (sec), $a=$ amber light time (sec), $l=$ lost time (sec), assumed to be $2 \mathrm{sec}$.

The unknown parameters $k, a$ and $C$ for each traffic signal were measured during April 2019. Three measurements were conducted for each traffic signal: during morning rush hour, evening rush hour and outside of rush hour, to account for expected time variations. Table 4 illustrates the final calculations of capacity for each urban road section.

Table 4: Estimation of Capacity for each urban road section

\begin{tabular}{lccc}
\hline Road Section & \multicolumn{3}{c}{ Capacity (pcu/h) } \\
& Morning Rush Hour & Outside of Rush Hour & Evening Rush Hour \\
\hline Main Arterial & 3540 & 3540 & 3374 \\
Secondary Arterial (Type 1) & 2562 & 2562 & 2562 \\
Secondary Arterial (Type 2) & 940 & 940 & 940 \\
Main Collector (Type 1) & 912 & 912 & 933 \\
Main Collector (Type 2) & 646 & 646 & 617 \\
Main Collector (Type 3) & 683 & 747 & 683 \\
\hline
\end{tabular}

\section{Calibration of Volume Delay Functions}

The estimation of the unknown parameters for the four most commonly used volume delay functions was performed using an algorithm written in Python, by minimizing the root mean square error (RMSE) between the observed and modelled speeds. The coefficient of determination was used as a supplementary performance indicator, so as to ensure the selection of the proper VDF for each road section. All functions were then reestimated and plotted using curve fitting tools in Matlab. It should be noted that the Akcelik function was not estimated for the road sections with varying values of capacity, since the parameter $c$ needs to be a constant variable, in order for the estimation to be feasible.

\subsection{Ring Road of Thessaloniki (type 1)}

The results in Table 5 and Figure 1 suggest that BPR fits the data well, followed by Davidson; whereas Conical and Akcelik functions underperform. BPR appears to be the best fit for the estimation of the average vehicle speeds, yielding $a=0.15$ as proposed by the literature for the typical BPR. The function is almost linear $(b=1.025)$, given that congestion was not reached. On the contrary, Conical and Akcelik showed poor fit to the 
data, as shown by the high RMSE and by the fact that both functions significantly underestimate the vehicle speeds for higher volumes.

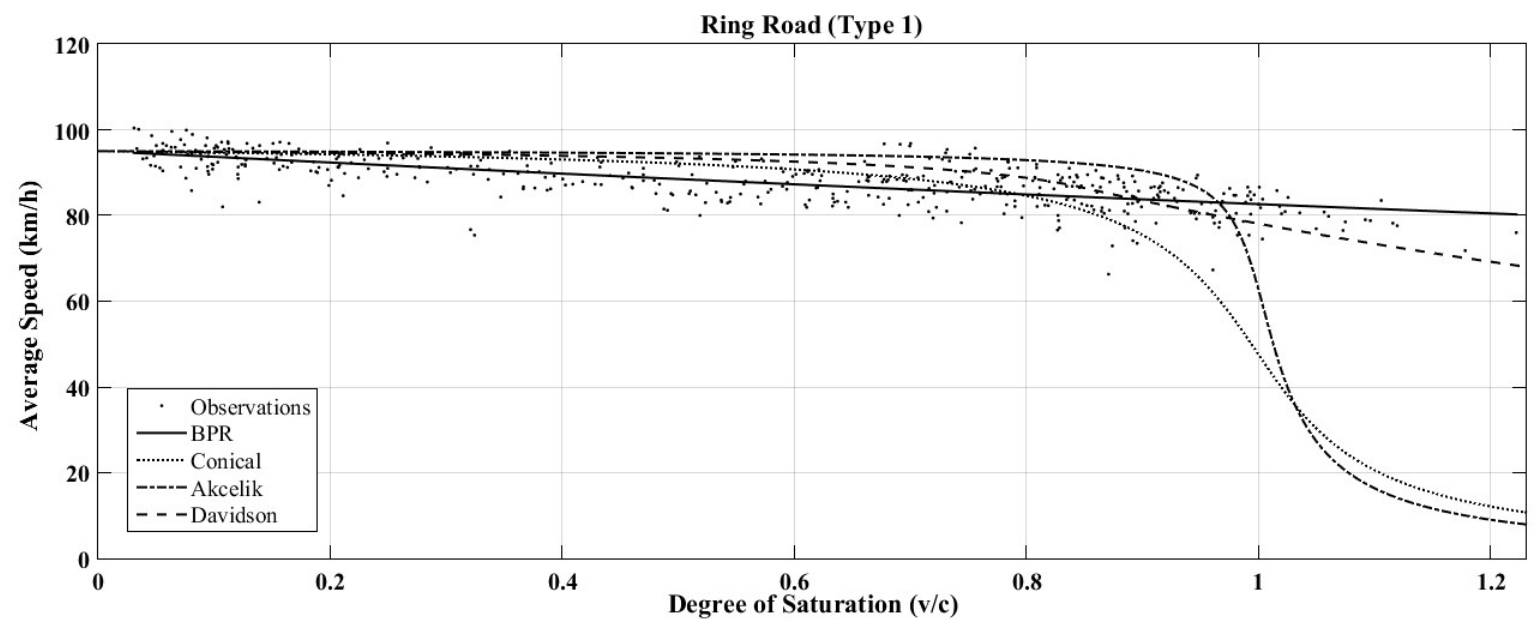

Figure 1: Fit of the data for the Ring Road (Type 1)

Table 5: Calibration of the VDFs for the Ring Road (Type 1)

\begin{tabular}{lccc}
\hline Function & Parameters & $R M S E$ & $R^{2}$ \\
\hline BPR & $\mathrm{a}=0.149$ & 3.965 & 0.489 \\
Conical & $\mathrm{b}=1.025$ & & \\
$\mathrm{a}=1.032$ & 13.842 & $\mathrm{~N} / \mathrm{A}^{*}$ \\
Akcelik & $\mathrm{b}=16.815$ & 12.987 & $\mathrm{~N} / \mathrm{A}^{*}$ \\
Davidson & $\mathrm{j}=0.329$ & 5.156 & 0.137 \\
\hline
\end{tabular}

*The algorithm did not converge

\subsection{Ring Road of Thessaloniki (type 2)}

Based on Table 6 and Figure 2, BPR remains the best fit for the observed vehicle speeds. Conical and Akcelik models underperform and intensely deviate from the observed field measurements for higher degrees of saturation, as shown in Figure 2. For this data set, the Davidson function provides a poor fit to the data, as derived from the higher RMSE compared to the calibration process for data set 1 . It should be noted that the BPR model is unable to depict the decline in vehicle speeds that correspond to the congested traffic conditions, since the curve is relatively flat $(b=0.765)$. The poor fit of the data could be attributed to the fact that the observed measurements were scattered, especially when congestion is reached $(v / c>1)$.

Table 6: Calibration of the VDFs for the Ring Road (Type 2)

\begin{tabular}{lccl}
\hline Function & Parameters & $R M S E$ & $R^{2}$ \\
\hline BPR & $\mathrm{a}=0.124$ & 5.088 & 0.277 \\
$\mathrm{~b}=0.765$ & 16.597 & \\
Conical & $\mathrm{a}=1.037$ & 16.219 & N/A* \\
Akcelik & $\mathrm{b}=15.014$ & 6.629 & N/A* \\
Davidson & $\mathrm{j}=0.169$ & N/A* \\
\hline
\end{tabular}

*The algorithm did not converge 


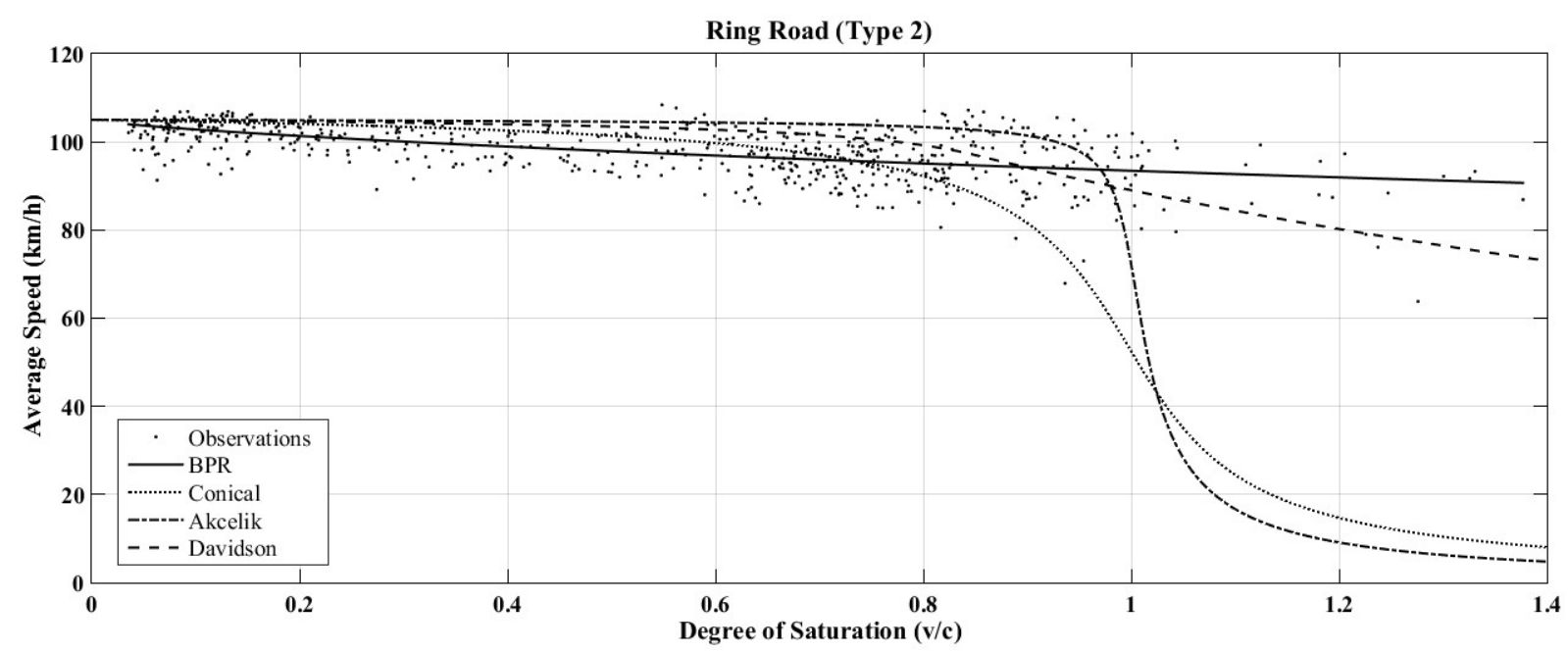

Figure 2: Fit of the data for the Ring Road (Type 2)

\subsection{Main Arterial}

As shown in Table 7 and Figure 3, the BPR, Conical and Davidson models perform in a similar way for the case of the Main Arterial. Since all functions have a relatively low RMSE, we consider that the BPR function could be characterized as the most suitable for the given road section, resulting in a slightly better performance compared to the other functions. It is worth mentioning, however, that congestion was not reached, during the field measurements, and, therefore, the decrease in speeds was minor, translating into relatively flat curves for all the estimated models.

Table 7: Calibration of the VDFs for the Main Arterial

\begin{tabular}{lccc}
\hline Function & Parameters & RMSE & $R^{2}$ \\
\hline BPR & $\begin{array}{l}\mathrm{a}=0.344 \\
\mathrm{~b}=1.020\end{array}$ & 2.632 & 0.395 \\
Conical & $\mathrm{a}=1.179$ & 2.907 & 0.261 \\
$\mathrm{~b}=3.787$ & 3.031 & 0.197 \\
Davidson & $\begin{array}{l}\mathrm{j}=0.141 \\
\mu=0.85\end{array}$ & 3 \\
\hline
\end{tabular}

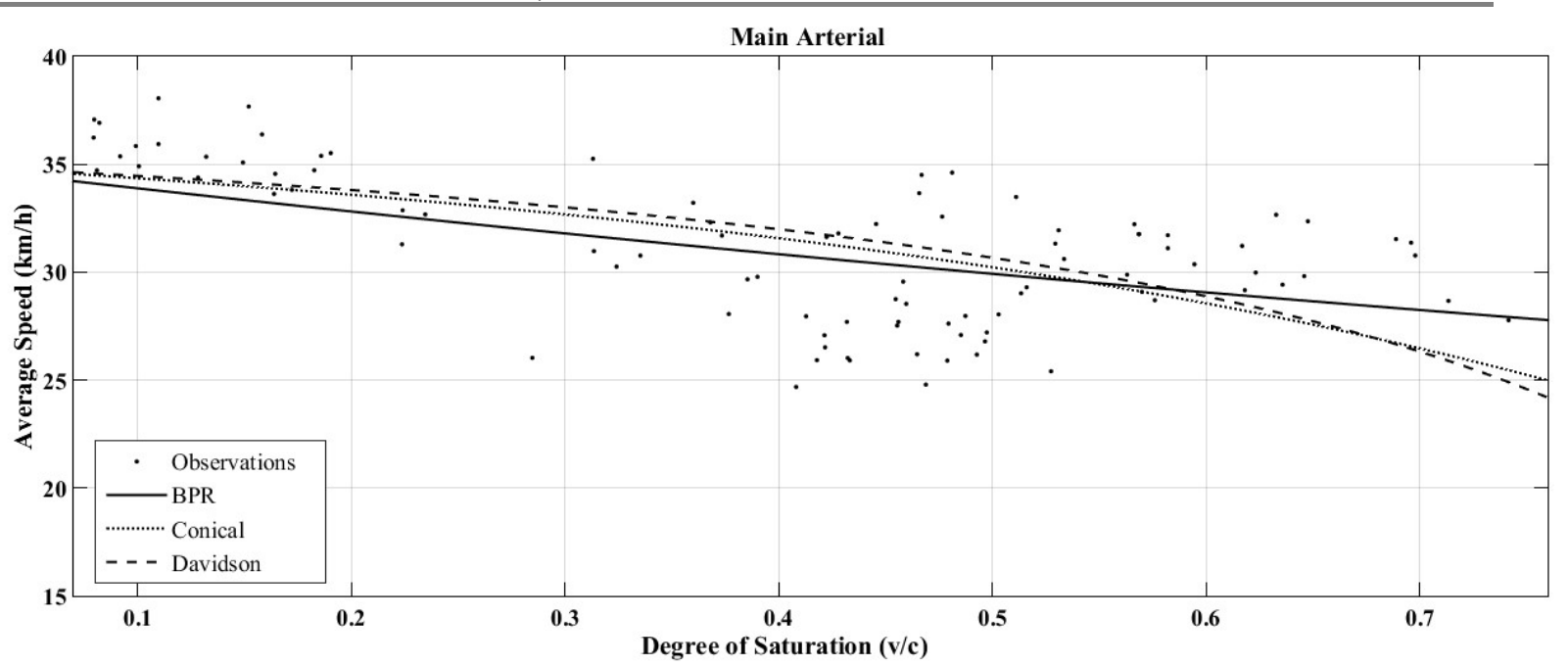

Figure 3: Fit of the data for the Main Arterial 


\subsection{Secondary Arterial (type 1)}

In the case of the first secondary arterial, all functions (with the exception of the Conical model) perform well, given the low values of RMSE and the outstanding high values of the coefficient of determination, which is higher than 0.8 for all three functions (Table 8). All three functions are capable of depicting the sharp decline in vehicle speeds for higher degrees of saturation, as illustrated in Figure 4. On the other hand, the calibration process for the Conical function resulted in a considerably higher RMSE and lower coefficient of determination and, therefore, the model leads to a noticeable overestimation of vehicle speeds for higher volumes. It should be noted that several outlier values were excluded from the calibration process for this data set, due to the existence of a pelican crossing which led to a sudden drop in speeds for low volumes.

Table 8: Calibration of the VDFs for the Secondary Arterial (Type 1)

\begin{tabular}{lccc}
\hline Function & Parameters & $R M S E$ & $R^{2}$ \\
\hline BPR & $\mathrm{a}=2.244$ & 4.274 & 0.862 \\
Conical & $\mathrm{b}=2.795$ & & \\
$\mathrm{a}=3.193$ & 6.289 & 0.690 \\
Akcelik & $\mathrm{b}=1.228$ & 4.609 & 0.834 \\
Davidson & $\mathrm{j}=16.95$ & 4.871 & 0.814 \\
\hline
\end{tabular}

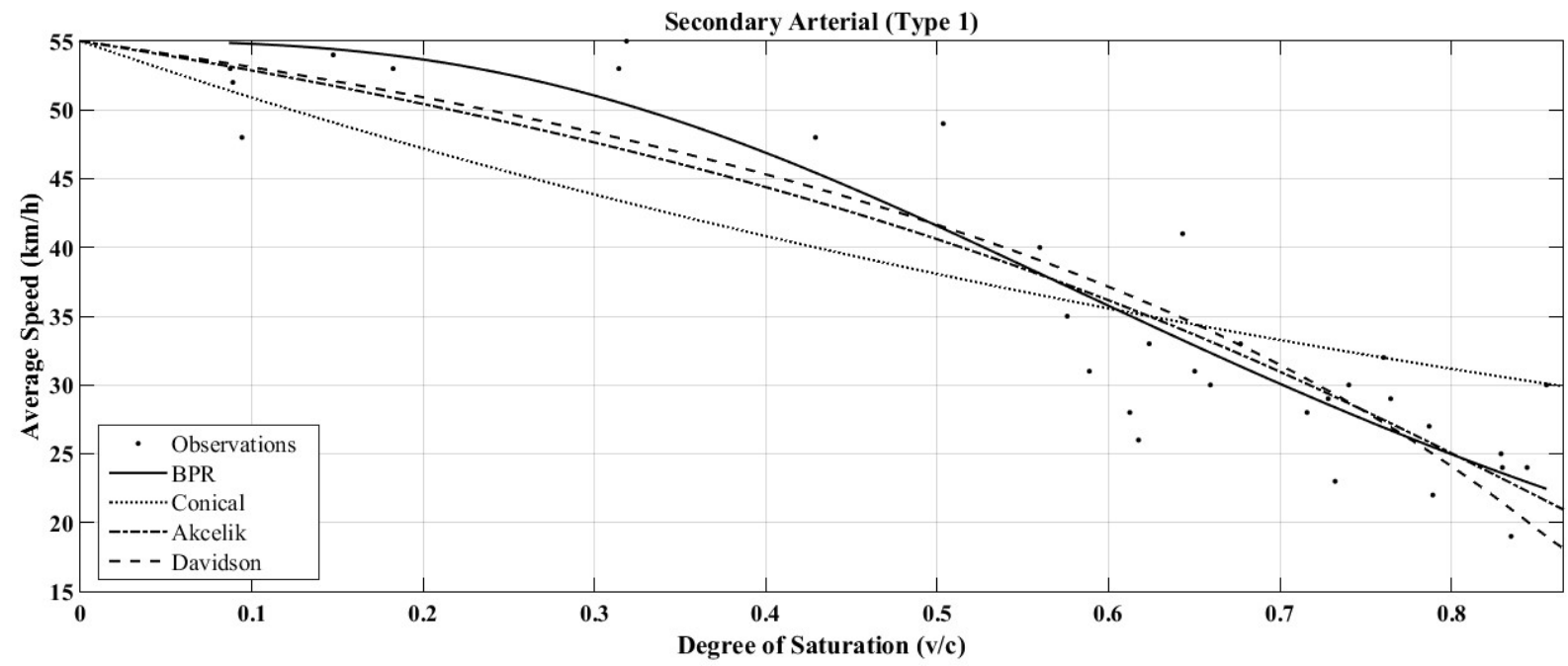

Figure 4: Fit of the data for the Secondary Arterial (Type 1)

\subsection{Secondary Arterial (type 2)}

As proposed in Table 9, the BPR curve fits the observed data well, followed by Akcelik, Davidson and, lastly, by Conical function. BPR is able to predict vehicle speeds with higher accuracy for the second secondary arterial, compared to the remaining models, despite the fact that the curve is relatively flat $(b<1)$. Conical function leads to a substantial overestimation of speeds (according to Figure 5) when the degree of saturation exceeds 0.2, whereas the opposite is true for Akcelik and Davidson, which marginally underestimate speeds for higher volumes. Capacity is rarely reached in this particular road section, and therefore the maximum volume to capacity ratio was slightly higher than 0.7 . Table 9: Calibration of the VDFs for the Secondary Arterial (Type 2) 


\begin{tabular}{lccc}
\hline Function & Parameters & RMSE & $R^{2}$ \\
\hline BPR & $\mathrm{a}=1.528$ & 2.985 & 0.857 \\
$\mathrm{~b}=0.985$ & $\mathrm{a}=6$ & 5.732 & 0.474 \\
Conical & $\mathrm{b}=1.1$ & 3.876 & 0.759 \\
Akcelik & $\mathrm{j}=15.251$ & 4.169 & 0.722 \\
Davidson & $\mathrm{j}=0.741$ & $\mu .85$ & \\
\hline
\end{tabular}

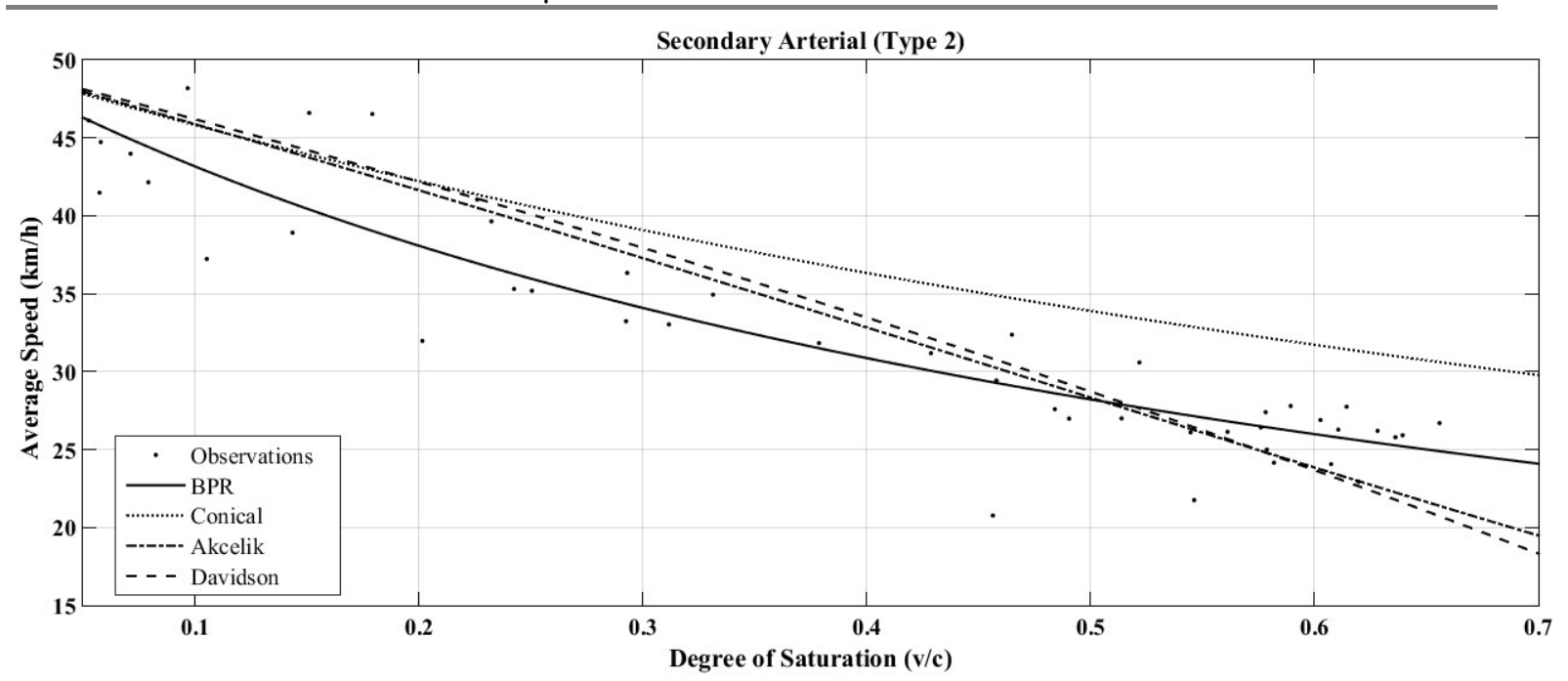

Figure 5: Fit of the data for the Secondary Arterial (Type 2)

\subsection{Main Collectors (group 1)}

The first group of main collectors consisted of the first and third main collectors, which yielded a free-flow speed of $45 \mathrm{~km} / \mathrm{h}$. BPR remains by far the best-performing model, as shown in Table 10 and Figure 6, despite being unable to depict the drop in speeds for higher volume counts. This could be attributed to the fact that the observations for this range of volumes are noticeably scattered, which implies that the road sections could perform differently for higher volumes. Davidson clearly deviates from the observations and results in the highest RMSE of approximately 3.94.

Table 10: Calibration of the VDFs for the Main Collectors (Group 1)

\begin{tabular}{lccc}
\hline Function & Parameters & $R M S E$ & $R^{2}$ \\
\hline BPR & $\mathrm{a}=0.638$ & 2.698 & 0.703 \\
Conical & $\mathrm{b}=0.862$ & & \\
$\mathrm{a}=1.759$ & 2.972 & 0.640 \\
$\mathrm{~b}=1.659$ & 3.944 & 0.366 \\
\hline
\end{tabular}




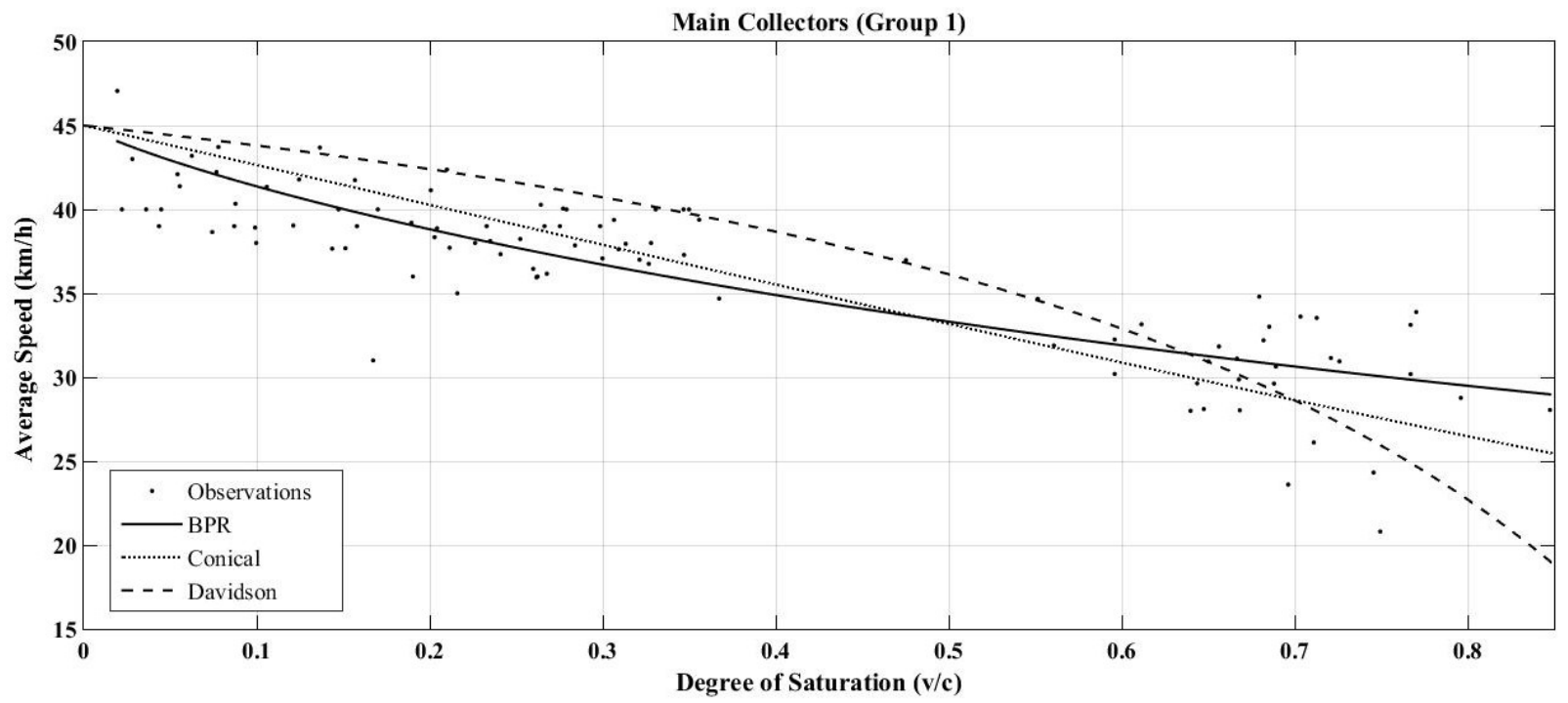

Figure 6: Fit of the data for the Main Collectors (Group 1)

\subsection{Main Collectors (Group 2)}

The second group of main collectors included the remaining second main collector, with a representative free-flow speed of $40 \mathrm{~km} / \mathrm{h}$. Based on Table 11 and Figure 7, BPR could be characterised as the most suitable function for this particular road section, leading to the lowest RMSE (2.58) among all the examined roads. On the contrary, the Davidson function underperforms by significantly overestimating the vehicle speeds for degrees of saturation lower than 0.8 .

Table 11: Calibration of the VDFs for the Main Collectors (Group 2)

\begin{tabular}{lccc}
\hline Function & Parameters & $R M S E$ & $R^{2}$ \\
\hline BPR & $\mathrm{a}=0.780$ & 2.580 & 0.860 \\
Conical & $\mathrm{b}=1.740$ & & \\
$\mathrm{a}=1.265$ & 2.930 & 0.820 \\
Davidson & $\begin{array}{l}\mathrm{b}=2.889 \\
\mathrm{j}=0.092 \\
\mu=0.85\end{array}$ & 3.968 & 0.662 \\
\hline
\end{tabular}

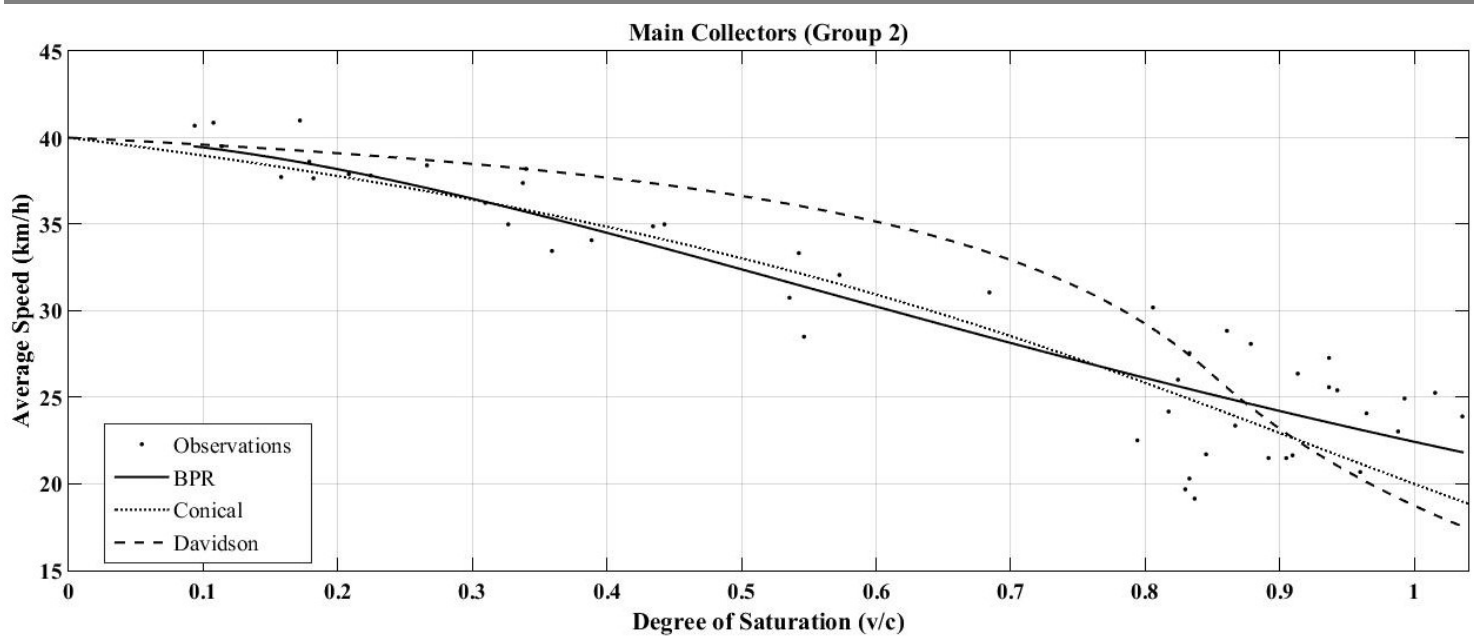

Figure 7: Fit of the data for the Main Collectors (Group 2) 


\section{Discussion}

The estimation process demonstrated the superiority of the updated BPR function, which was proved to be the best fit for the prediction of vehicle speeds across a wide variety of different road sections. This result further validates the widespread use of the BPR function in the trip assignment process, as previously proposed by literature (Skabardonis and Dowling, 1997; Kucharski and Drabicki, 2017; Marquez, 2014). The RMSE ranged from 2.58 to 5.09 indicating high accuracy in the prediction of vehicle speeds across all the examined facilities. Surprisingly, the coefficient $a$ equals 0.15 for the first data set of the Ring Road (freeway), which is the suggested value of $a$ for the typical BPR function (Mtoi and Moses, 2014; Marquez, 2014). On the contrary, the values of the coefficient $b$ are significantly lower than those used in other countries. For the Ring Road of Thessaloniki, $b$ was shown to be noticeably lower than the values proposed for Denmark (Nielsen and Jorgensen, 2008), for Australia (Bagherian, Hichman and Tavassoli, 2017) and for Paris (Cohen and Zhang, 2002), which were estimated to be 4 , 2.64 and 7.15, respectively. Further comparison for the selected urban sections is not feasible, since capacity was estimated differently and the analysis covered the whole road section, instead of focussing on each lane.

The Conical model was found to be a good fit for the prediction of vehicle speeds in main collectors. However, the coefficient $b$ is significantly lower than 5 across all undivided arterials and, therefore, differs from the value proposed in literature (Davis and Xiong, 2007) for this particular road type. Conical function clearly underperforms in the case of the Ring Road, which contradicts the results by Mtoi and Moses (2014), whose study showed lower RMSE for freeways and higher for signalized arterials, for the Conical function.

In contrast, their results for the Akcelik function are further validated in our study, since the Akcelik model clearly underperformed for freeways but provided a better fit for all the signalized arterials in which the estimation was feasible. Furthermore, the analysis yielded higher values for the coefficient $j$ for arterial roads without cordination compared to freeways, as suggested by Akcelik (Mtoi and Moses, 2014; Dowling, Singh and Cheng, 1998). The Akcelik function was found to predict the vehicle speeds with higher accuracy in comparison with the Modified Davidson function, for all the examined urban sections. Despite the existence of the coefficient $\mu$, which allegendly results in a better representation of the traffic conditions, the Davidson function clearly underperformed, compared to the BPR model.

The calibration process revealed substantial differences in the values of the unknown coefficients for all volume delay functions across the different facility types, which verifies the significance of further dividing the arterial roads in major and minor arterials, which is not, however, adopted widely in practice. Moreover, the coefficients were shown to differ significantly from the values proposed in literature, suggesting the necessity of the re-estimation of the unknown parameters with locally derived data to allow for a better representation of the driver behavior in the examined area. Finally, the importance of an accurate estimation of the free-flow speed was validated, given that the calibration process yielded different results for road sections of the same facility type with a minor difference in free-flow speed of $5 \mathrm{~km} / \mathrm{h}$. 


\section{Conclusions}

The precise determination of volume delay functions plays a critical role in the traffic assignment process. This study aimed to calibrate the most commonly used volume delay functions for several road sections of the city of Thessaloniki, based on recent real-world traffic data, and to define the most suitable function for each facility type. Undoubtedly, the BPR model resulted in a satisfactory goodness-of-fit for both interrupted and uninterrupted facilities and could be used for the prediction of vehicle speeds in the examined road sections.

This research,however, is subject to several limitations. The estimation process was based on pre-processed data of hourly speeds and volumes. It would have been desirable to have access to the time mean speed of each vehicle, in order to exclude outlier values. Furthermore, the data concerned the whole road section instead of focussing on each lane, which would allow to account for the differences in driver behavior between the lanes, especially for the case of the Ring Road. In several road sections congestion was not reached and, therefore, the calibration led to flat curves which can not simulate the traffic conditions when volumes exceeds capacity. Finally, 15-minute time periods would result in considerably smaller errors in the estimation of the volume delay functions, especially for the case of the Akcelik function.

Future directions of this research could focuss on real-world data captured through floating-car techniques, given that space-mean speed has been shown to increase the accuracy of the predicted vehicle speeds. The emergence of autonomous vehicles and their expected widespread use are expected to alter traffic conditions and have a radical effect on driver behavior. As a result, the proposed curves need to be re-evaluated, to take in to account the occuring changes in traffic. The application of big data techniques in the field of transportation planning seems promising, since real-time data capturing incidents and unexpected events could potentially lead to a more sustainable and effective traffic distribution, through dynamic traffic assignement models.

\section{References}

Antoniou, C. and Spyropoulou, I. (2015) Principles of transportation engineering and simulation. Kallipos. Available at: https://repository.kallipos.gr/pdfviewer/ web/viewer.html?file=/bitstream/11419/5793/2/TFT-fullPDF-

$\%$ ce $\% 9 a \%$ ce $\% 9 f \%$ ce $\% a 5 . p d f$

Bagherian, M., Hickman, M. and Tavassoli, A. (2017) "Considering the impact of precipitation on the accuracy of delay-function parameters", Proceedings from the $39^{\text {th }}$ Australasian Transport Research Forum.

Barka, R. E. (2019) Calibration and evaluation of nonlinear speed volume functions for different types of road sections. Diploma Thesis, Aristotle University of Thessaloniki, Thessaloniki.

Chepuri, A., Raju, N., Bains, M.S., Arkatkar, S. and Joshi, G. (2018) "Examining Performance of an Urban Corridor Using Microscopic Traffic Simulation Model under Mixed Traffic Environment in India”, European Transport- Transporti Europei, (69), pp. 1-21 Available at: http://www.istiee.unict.it/europeantransport /papers/N69/P02_69_2018.pdf

Cohen, S. and Zhang, M. Y. (2002) "New speed flow relationships for road network planning in the Paris Region", Proceedings from the Conference on Traffic and Transportation Studies, ICTTS, pp. 389-396. doi: 10.1061/40630(255)54. 
Davis, G. A. and Xiong, H. (2007) Access to destinations: travel time estimation on arterials. Minnesota: Minnesota Department of Transportation.

Dhulipala, S., Kedia, A.S. and Katti, B.K. (2017) "Multi-route choice modelling in a metropolitan context: A comparative analysis using Multinomial Logit and Fuzzy Logic based approaches", European Transport- Transporti Europei, (79), pp. 1-17 Available at: http://www.istiee.unict.it/sites/default/files/files/Paper\%204\%20n\%2079.pdf

Dowling R. G., Singh, R. and Cheng, W. W. (1998) "Accuracy and performance of improved speed-flow curves", Transportation research record, (1646), pp. 9-17. doi: $\underline{10.3141 / 1646-02}$

Dutta, M. and Ahmed, M.A. (2019) "Capacity of Saturated Streams at Manually Controlled Intersections", European Transport- Transporti Europei, (73), pp. 1-22 Available http://www.istiee.unict.it/europeantransport/papers/N73/P06_73_2019.pdf

He, N. and Zhao, S. (2013) "Discussion on Influencing Factors of Free-flow Travel Time in Road Traffic Impedance Function", Procedia - Social and Behavioral Sciences, 96 (1), pp. 90-97. doi: 10.1016/j.sbspro.2013.08.013

Huntsinger, L.F. and Rouphail, N. M. (2011) "Bottleneck and queuing analysis: calibrating volume-delay functions of travel demand models", Transportation research record, (2255), pp. 117-124. doi: 10.3141/2255-13

Kimber, R.M. , McDonald, M. and Hounsell, N.B. (1986) The prediction of saturation flows for road junctions controlled by traffic signals. Crowthorne: Transport and Road Research Laboratory.

Kucharski, R. and Drabicki, A. (2017) "Estimating Macroscopic Volume Delay Functions with the Traffic Density Derived from Measured Speeds and Flows", Journal of Advanced Transportation, 2017 (1). doi: 10.1155/2017/4629792

Marquez, L. (2014) "Conical and the BPR Volume-Delay Functions for Multilane Roads", Boletín Técnico, 54 (3), pp. 14-24.

Martin, W. A. and Mcguckin, N.A. (1998) Travel Estimation Techniques for Urban Planning. Washington DC: National Academy Press.

Moses, R., Mtoi, E., Ruegg, S. and McBean, H. (2013). Development of speed models for improving travel forecasting and highway performance evaluation. Florida: Department of Transportation.

Mtoi, E. and Moses, R. (2014) "Calibration and Evaluation of Link Congestion Functions: Applying Intrinsic Sensitivity of Link Speed as a Practical Consideration to Heterogeneous Facility Types within Urban Network", Journal of Transportation Technologies, 4, pp. 141-149. doi: 10.4236/jtts.2014.42014

Nielsen, O.A. and Jørgensen, R.M. (2008) "Estimation of speed-flow and flow-density relations on the motorway network in the greater Copenhagen region", IET Intelligent Transport Systems, 2 (2), pp. 120-131. doi: 10.1049/iet-its:20070024

Nobel, D. and Yagi, S. (2017) "Network Assignment Calibration of BPR Function: A Case Study of Metro Manila, the Philippines", Journal of the Eastern Asia Society for Transportation Studies, 12, pp. 598-615. doi: 10.11175/easts.12.598

Oskarbski, J., Jamroz K., Smolarek, L., Zawisza, M. and Zarski, K. (2017) “Analysis of possibilities for the use of volume-delay functions in the planning module of the tristar system", Transport Problems, 12 (1), pp. 39-50. doi: 10.20858/tp.2017.12.1.4

Rose, G. (1994) "Impacts of estimation errors in travel time functions", Transportation Planning and Technology, 18 (4), pp. 249-262. doi: 10.1080/03081069408717548 
Rose, G., Taylor, M.A.P. and Tisato, P. (1989) "Estimating travel time functions for urban roads: options and issues", Transportation Planning and Technology, 14 (1), pp. 63 82. doi: $10.1080 / 03081068908717414$

Seetharaman, P., Errampalli, M., Senathipati, V., Shukla, A. and Gangopadhyay, S. (2011) "Modeling time mean speed and space mean speed for heterogeneous traffic conditions", Transportation Planning and Technology, 34 (8), pp. 823-838. doi: 10.1080/03081060.2011.613593

Skabardonis, A. and Dowling, R. (1997) "Improved speed-flow relationships for planning applications”, Transportation research record, (1572), pp. 18-23. doi: 10.3141/1572$\underline{03}$

Spiess, H. (1990) “Technical Note-Conical Volume-Delay Functions", Transportation Science, 24 (2), pp. 153-158. doi: 10.1287/trsc.24.2.153

Stevens, R. S., Barkley, A.S. and Miller, J.S. (2017) "Impact of Site-Specific Data on the Accuracy of Volume Delay Functions", Proceedings from the $96^{\text {th }}$ annual meeting of Transportation Research Board, Washington DC.

Subbarao, S.S.V. and Krishnarao, K.V. (2016) "Activity based approach to travel demand modelling: An overview", European Transport- Transporti Europei, (61). Available at: http://www.istiee.unict.it/europeantransport/papers/N61/P06_61_08_2016.pdf

Suh, S., Park, C.H. and Kim, T.J. (1990) "A highway capacity function in Korea: measurement and calibration”, Transportation Research Part A: General, 24 (3), pp. 177-186. doi: 10.1016/0191-2607(90)90055-B

Transportation Research Board. 2010. Highway Capacity Manual 2010. $5^{\text {th }}$ ed. Washington DC: Transportation Research Board. 